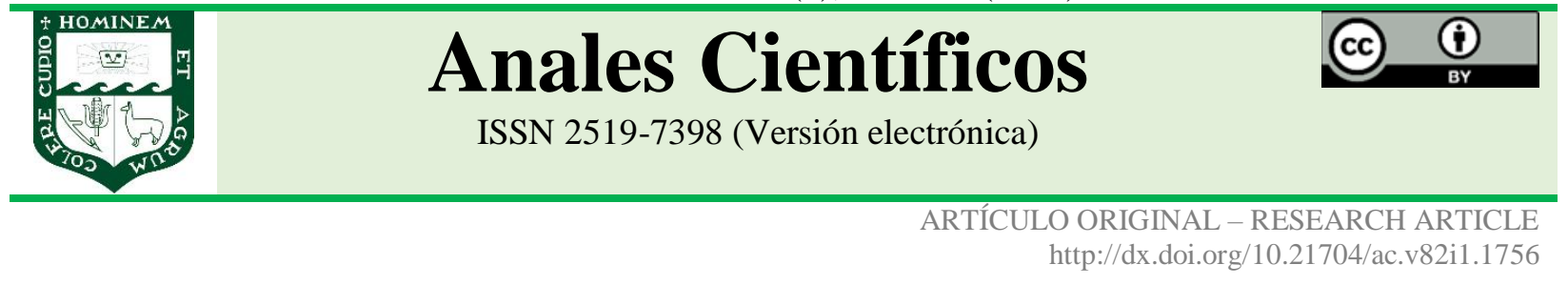

\title{
ANÁLISIS DE ROTURA DE DIQUE DURANTE EL HURACÁN KATRINA 2005
}

\author{
Analysis of dam breakage during Hurricane Katrina 2005 \\ Alfonso Armando Cerna-Díaz ${ }^{1 *}$; Alfonso Cerna-Vásquez ${ }^{2}$ \\ 1 Investigador independiente, Denver- Colorado, EEUU. \\ ${ }^{2}$ Facultad de Ingenieria Agricola, Universidad Nacional Agraria La Molina, La Molina 15024. Lima, Perú \\ E-mail: alcernadiaz@gmail.com
}

Recibido: 24/09/2019; Aceptado: 12/01/2021; Publicado: 31/08/2021

\begin{abstract}
Hurricane Katrina was the costliest and one of the deadliest natural disasters in the history of the United States. It resulted in catastrophic loss of life and left thousands without homes. Levee breaches surrounded the city of New Orleans as a result of the massive storm surge. Many levee failures occurred including the 17 th Street Canal, Industrial Canal, London Avenue Canal, and the Inner Harbor Navigation Canal (IHNC). This report focuses on investigating the mode of failure of the south breach on the east bank of the Inner Harbor Navigational Canal. Computer software Seep/W and Slope/W from Geostudio were used to estimate levee transient flow and critical stability case using Spencer's rigorous method. Results indicate that piping failure mechanism did not occur as computed hydraulic gradients were lower than the critical value. Furthermore, factor of safety against semirotational failure is 1.24. Finally, from erosion photos and a semi-empirical solution estimate, a $3 \mathrm{ft}$ of erosion at the toe of the levee occurred that triggered a factor of safety against overturning of 0.94 . Additionally, for the same erosion condition, a factor of safety of 1.42 was obtained against translational failure mechanism. Based on the results, it is concluded that the critical mode of failure due to erosion was overturning.
\end{abstract}

Keywords: Critical failure surface; transient flow; soil profile; scour; crack; stability;

\section{RESUMEN}

El huracán Katrina fue el desastre natural más costoso y uno de los más mortales en la historia de los Estados Unidos. Muchos diques que circundan la ciudad de Nueva Orleans fueron afectados como resultado del evento de tormenta masivo. Se produjeron muchas fallas en los diques, incluidos el canal de la calle 17, el canal industrial, el canal de la avenida London y el canal de navegación del puerto interior (IHNC). Este trabajo de investigación tuvo como objetivo central evaluar el modo de falla del sector sur en la orilla este del dique de tierra IHNC. Se utilizaron programas de cómputo Seep/W y Slope/W de Geostudio para estimar el flujo transiente y estabilidad del dique para determinación del evento crítico. El análisis de estabilidad se realizó mediante el método riguroso de Spencer. Los principales resultados encontrados nos indican que las falla por tubificación no se ha producido debido a que el gradiente máximo de salida es menor que el gradiente crítico del suelo. También se pudo encontrar que el factor de seguridad para la estabilidad semi-rotacional del dique es de 1,24. Finalmente; a partir de fotos de erosión y métodos semiempiricos se estimó 3 pies de socavación al pie del dique, lo que permite obtener un factor de seguridad contra el vuelco F.S = 0,94, obteniéndose además que para la misma socavación, la estabilidad por traslación fue controlado, obteniéndose un FS = 1,42. En base a los resultados obtenidos, se concluye que el modo crítico de falla debido a la erosión es el de volcamiento.

Palabras clave: Falla crítica; infiltración transiente; perfil del suelo; erosión; grieta; estabilidad; tubificación 


\section{Forma de citar el artículo (Formato APA):}

Cerna-Diaz, A., \& Cerna-Vasquez. (2021). Análisis de rotura de dique durante el huracán Katrina 2005. Anales Científicos. 82(1), 130-140. http://dx.doi.org/10.21704/ac.v82i1.1756

Autor de correspondencia (*): Alfonso Cerna-Diaz. Email: alcernadiaz@gmail.com

(c) Los autores. Publicado por la Universidad Nacional Agraria La Molina.

This is an open access article under the CC BY

\section{INTRODUCCIÓN}

El estudio de la rotura de dique durante el huracán Katrina es una investigación que permite explicar desde el punto de vista geotécnico, las causas que originaron el desastre natural más costoso y uno de los más mortales en la historia de los Estados Unidos. Al menos 1,833 personas perdieron la vida, y los daños causados por el huracán se estiman en más de $\$ 80$ mil millones. El huracán originó rupturas en 50 diques de contención; lo que significó, aproximadamente que el $80 \%$ de la ciudad de Nueva Orleans se inundara a profundidades de hasta 15 pies. La tormenta ciclónica fue canalizada por la vía fluvial intracostera del golfo y la salida del golfo del río Mississippi y originó daños en dos lugares en el extremo este del IHNC.

La multiplicidad de los tipos de suelo de origen residual, que son encontrados en los EEUU y en otras regiones del mundo, combinado con el efecto inesperado que causan en las estructuras los fenómenos naturales como sismos, huracanes y otros, hacen cada vez más importante y necesario disponer de un buen conocimiento geotécnico acerca del medio en donde se cimentarán diversas obras de ingeniería.

La aún falta de conocimiento del subsuelo ha producido, en numerosos casos, fallas importantes en obras e incluso pérdidas de vidas. Son frecuentes los problemas asociados con asentamientos importantes, empujes y baja capacidad de soporte, entre otros. En la Ingeniería geotécnica cada reporte o investigación de hechos como lo sucedido con los efectos del huracán Katrina es de significancia científica.

La ciudad de Nueva Orleans construida originalmente sobre antiguos cursos de agua y diques naturales que se ubicaban sobre el nivel del mar (IPET, 2006), tiene como infraestructura de defensa el dique IHNC, el cual es un dique de tierra con muro de tablestacas cuya función es de reducir la infiltración en los materiales subyacentes. Aunque hubo dos fallas en la orilla este del IHNC, la del sector sur de aproximadamente 850 pies de ancho fue la más catastrófica y resultó ser la de mayor daño (Sills et al., 2008; Bea, 2008; Seed et al., 2007). El modo de falla del sector sur en el extremo este no fue inmediatamente identificado. El objetivo principal de la investigación consistió en evaluar el modo de falla del sector sur en la orilla este del dique de tierra IHNC y para ello los autores realizaron múltiples análisis utilizando el software GeoStudio, teorías de presión de tierras y el método propuesto por Briaud et al. (2008), para investigar los diferentes modos de falla crítica en los suelos de soporte, que podrían haber originado el desastre.

\section{MATERIALES Y MÉTODOS}

Se presenta el desarrollo de la investigación mediante la descripción de los suelos que conforman la zona en estudio, mediante sus propiedades geológicas, geotécnicas, las características de los diques naturales, zonas de inundación, de relleno, excavaciones y muros diseñados para proteger la zona en estudio. Se analiza la infiltración transiente en base al hidrograma del evento, modelando también la tormenta como una condición límite cambiante. Los análisis de estabilidad para las condiciones límites planteadas en la presente investigación, se realizan mediante las diferentes aplicaciones existentes para resolver problemas geotécnicos, tales como, el software GeoStudio, el método propuesto por Briaud et al. (2008) para determinar la erosión por socavación, el fenómeno de tubificación se analizó utilizando el programa de infiltración GeoStudio Seep/W (Geo-Slope, 2012a) y la estabilidad semi-rotacional se evaluó utilizando el método de Spencer en el software Slope/W (GeoSlope, 2012b). Estos métodos permiten modelar mejor los cambios en la estabilidad durante el desarrollo del Huracán Katrina.

\section{Antecedentes geológicos}

La ciudad de Nueva Orleans fue construida originalmente sobre antiguos cursos de agua y 
diques naturales que se ubicaban sobre el nivel del mar (IPET, 2006). Sin embargo, el desarrollo posterior de la ciudad, requirió la colocación de un relleno sobre un pantano blando para elevar el terreno circundante. Las elevaciones superficiales en Nueva Orleans generalmente oscilan entre $+/-10$ pies sobre el nivel del mar. Debido a que aproximadamente la mitad de Nueva Orleans está por debajo del nivel del mar, se necesita un bombeo constante para evitar que partes de la ciudad se sumerjan. Como resultado, los antiguos materiales del pantano cerca de la superficie son altamente compresibles cuando se cargan o bombean, originando asentamientos continuos debido a la desecación que origina el bombeo (Dunbar et al., 2008).

\section{Geología regional}

La geología de la región de Nueva Orleans está muy influenciada por el delta del río Mississippi, este río desemboca en el Golfo de México. La superficie del suelo en Nueva Orleans generalmente está constituida de un relleno artificial de hasta 15 pies de espesor. Antes de urbanizar el área, los antiguos pantanos de cipreses muy blandos y altamente compresibles dominaban la superficie, y se necesitaba de un bombeo intensivo y la colocación de relleno antes de iniciar cualquier construcción. Estos materiales de pantano se denominan "Arcilla orgánica" en este informe. La capa superior tiene mayor presencia de arcilla orgánica que puede deberse a la desecación por causas naturales y de bombeo. Debajo de los pantanos existe aproximadamente $70-80$ pies de unidades del Holoceno de espesor variable. Estas unidades varían en tamaño de grano, principalmente del tamaño de arena a arcilla, y se depositaron en: la bahía, cerca del golfo de la costa, intradelta, playa relicta, lacustre y entornos interdistributarios (IPET, 2006). Los suelos del Holoceno están fundados sobre depósitos pleistocenos más antiguos.

\section{Perfil del suelo}

Se obtuvo una sección transversal del sector sur de la ciudad, lugar afectado por el huracán Katrina (Figura 1). Con base a la evaluación preliminar, se determinó que las unidades subyacentes a la arcilla interdistributaria no tendrían esencialmente ningún impacto en los análisis. Por lo tanto, los análisis contenidos en este informe solo consideraron las unidades de arcilla interdistributaria y superiores. Estas unidades se describen brevemente a continuación. Las propiedades del material utilizadas para el análisis se detallan en la sección 3. El perfil del suelo en el sector sur contiene las siguientes unidades: Relleno (varía en grosor y tipo), arcilla orgánica superior, Arcilla orgánica baja $\mathrm{y}$ arcilla interdistributaria (ID)

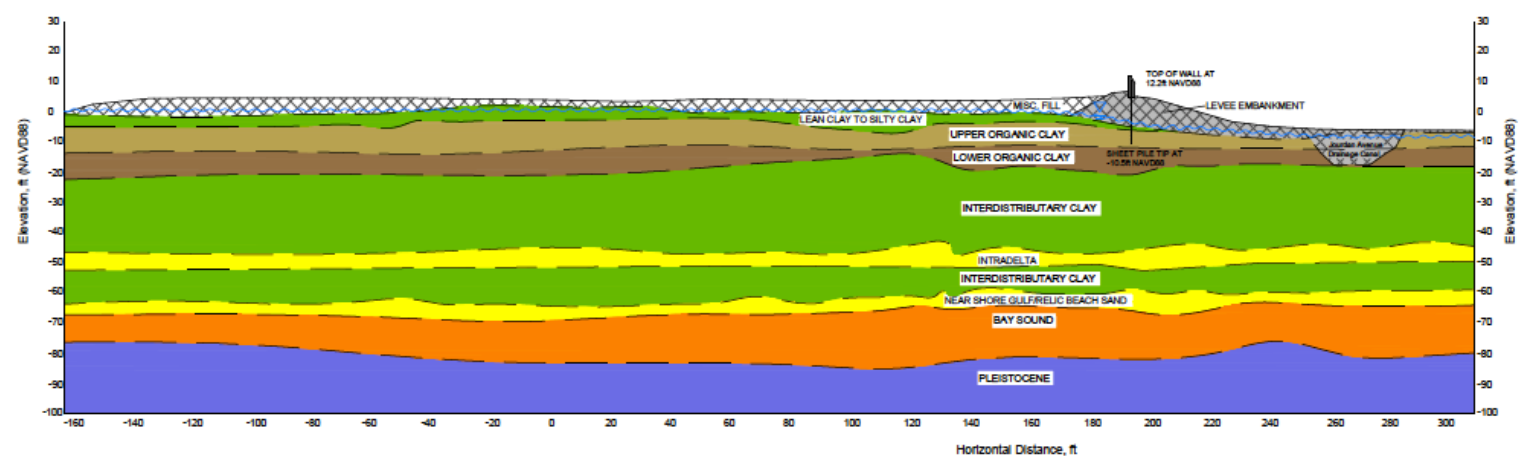

Figura 1. Perfil del Suelo de la Rotura del Dique en la Zona Sur (IPET, 2006).

a. Dique Relleno. Por encima de los suelos naturales, se consideró cinco materiales de relleno diferentes en el análisis: "Shell", Relleno de tierra, Excavación SM-5 (ubicada adyacente al IHNC y no se muestra en la Figura 1), Relleno de dique y Canal Jourdan Avenue (JAC). El material denominado "Shell" presenta componentes de origen marítimo y está entre la excavación SM-5 y la parte húmeda del dique; Teniendo una mayor permeabilidad en comparación con los rellenos dique y en la zona aguas abajo del embalse. El "Relleno del dique" es el cuerpo del dique, 
y el relleno en la zona aguas abajo está al costado del terraplén. Los efectos de SM-5 y JAC se presentan en la sección 4. Las propiedades del suelo se presentan en la sección 3.

b. Capas de arcilla orgánica. Las capas de "Arcilla orgánica" consisten en una capa superior más fuerte, denominada "Arcilla orgánica superior", y una capa subyacente más débil y permeable denominada "Arcilla orgánica superior ". Estas capas de arcilla orgánica son las capas más débiles y permeables del sistema. Las propiedades de los suelos se discuten en la sección 3.

c. Arcilla interdistributaria. La arcilla ID es una arcilla que se aproxima a un estado de una normalmente consolidada que subyace a las capas de arcilla orgánica. Esta capa representa un borde relativamente impermeable de alta resistencia en la base de la arcilla orgánica. Las propiedades de esta arcilla se discuten en la sección 3.

\section{Consideraciones}

Diseño del muro de sección I

El muro del dique consiste en una pared de concreto sobre la superficie del suelo con una tablaestaca que se extiende desde la arcilla orgánica superior hasta una elevación de aproximadamente -11 pies. El muro no penetra en el fondo de la arcilla orgánica superior a lo largo del sector sur. La elevación superior de la pared es de aproximadamente $+12,5$ pies. En los análisis mediante el Seep/W y Slope/W, el muro se modela como un elemento impermeable de 1 pie de ancho con una resistencia mucho mayor que la del suelo.

\section{Evento de Tormenta}

El evento de tormenta inusualmente grande se originó por el efecto de embudo entre las cercanías al Canal Intracostero del Golfo y la salida del Golfo del río Mississippi. Originó niveles de agua de más de 14 pies. El Hidrograma para el IHNC, que se muestra en la Figura 2, se utilizó para el modelamiento de la infiltración y estabilidad de los taludes.

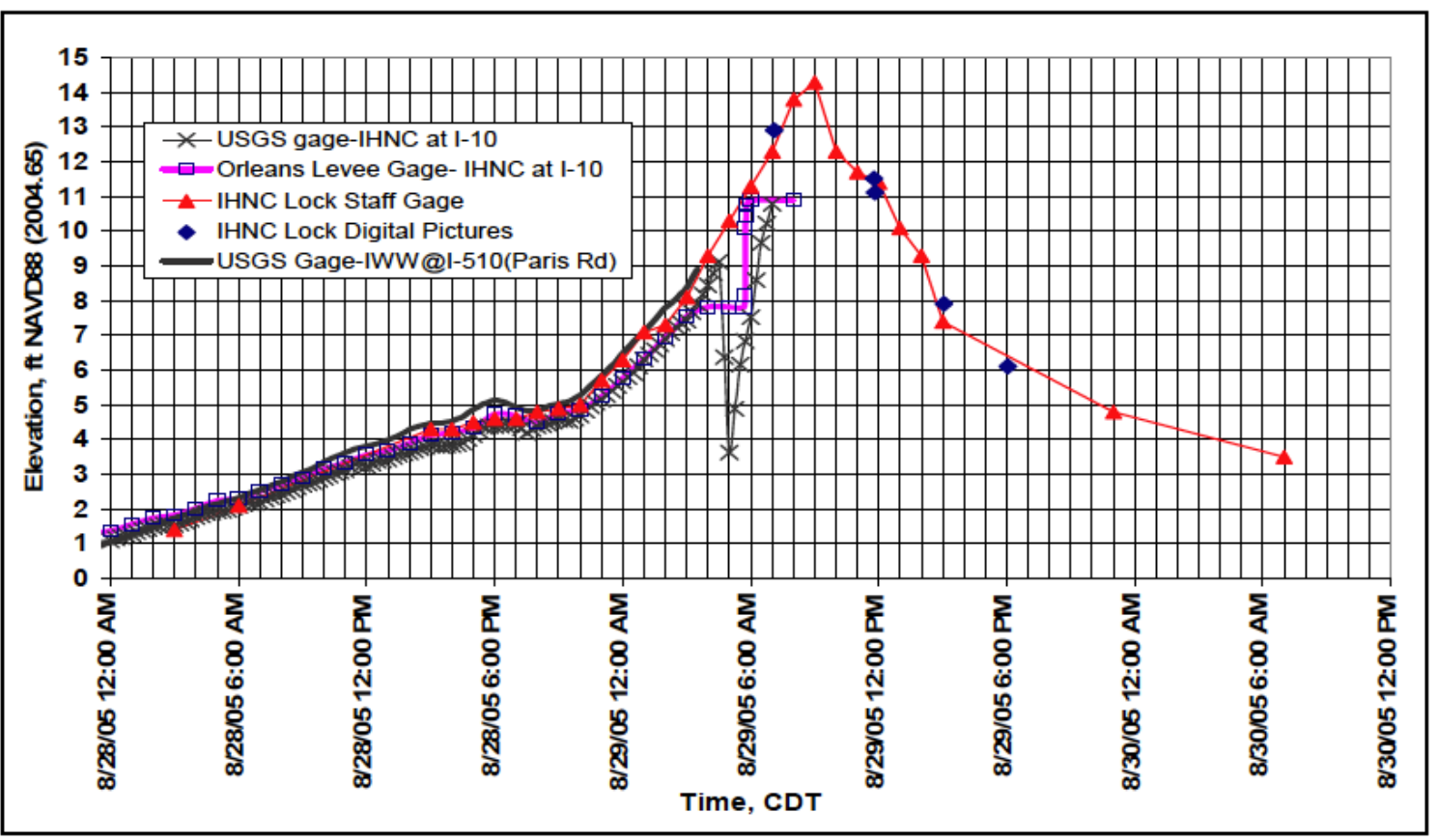

Figura 2. IHNC información hidrográfica (IPET, 2006).

\section{Rebalsamiento del Dique}

El agua rebalsó al dique de dos formas: Primero por la acción de las olas, segundo por la permanente sobrecarga luego de que la tormenta llegara al techo del dique (Rogers, 2007). El exceso de agua erosionó el suelo de la base del dique, lo que condujo a la pérdida de estabilidad. Briaud et al. (2008) propuso un método para determinar la erosión por socavación. Este método se discutirá en la sección 3. 
Formación de la grieta entre el muro y el dique Cuando ocurrió el desembalse del dique, inmediatamente se inició la erosión el pie del muro creando un estado de inestabilidad permitiendo la creación de una grieta entre el muro y el dique en el lado aguas arribas del dique (Duncan et al., 2008). Este fenómeno, exacerbo aún más la inestabilidad que originó la rotura del dique, generándose la inundación. Los análisis contenidos en este documento suponen que la formación de esta grieta en el sector de aguas arriba se inicia cuando la tormenta alcanzó el punto medio entre la superficie del suelo y la parte superior del muro, a una altura de 9,5 pies, y se extendió a lo largo del muro de tablestacas. Se asume conservadoramente que la grieta se forma desde la base del muro. Con la formación de la grieta, la estabilidad disminuye de tres formas: 1) la estabilidad al corte disminuye al reducir la dimensión de la superficie de falla semirotacional; 2) la fuerza sobre la pared aumenta al incluir una fuerza hidrostática del agua, y 3) los gradientes aumentan al reducir la trayectoria de infiltración.

\section{- Rellenos y excavaciones}

El Canal Jourdan Avenue (JAC) pasa por el relleno del dique aguas abajo al muro. El canal es una alcantarilla de drenaje para la escorrentía de agua. El relleno para el canal es una arcilla menos permeable que los suelos de arcilla orgánica circundantes. Esto hace que cualquier infiltración debajo del dique acumule presiones al llegar al canal. Estas presiones se investigan en el análisis de infiltración / tuberías en la sección 3. La excavación SM-5, cerca del IHNC, se rellenó en el momento de la tormenta. El relleno se modeló como una permeabilidad más alta que el relleno del dique y la arcilla orgánica.

\section{Cronograma de eventos}

Para realizar un análisis de infiltración transiente, el evento de tormenta se modela como una condición límite cambiante, que sigue al cambio de la tormenta en función del tiempo. Otras condiciones que cambian con el tiempo son los espacios originados en la rotura del sector de inundación y la erosión en la base de tierra del muro de sección I. Los análisis se realizan bajo estas condiciones cambiantes para modelar mejor los cambios en la estabilidad durante el huracán. La línea de tiempo utilizada para el análisis se da en la Tabla 1.

Tabla 1. Datos en el tiempo.

\begin{tabular}{lll}
\hline Fecha & Hora & \multicolumn{1}{c}{ Descripción } \\
\hline $8 / 28 / 2005$ & $12: 00 \mathrm{am}$ & Inicio de la tormenta \\
8/29/2005 & $2 \mathrm{am}-4 \mathrm{am}$ & Aceleración máxima de la tormenta \\
8/29/2005 & $4: 00 \mathrm{am}$ & Se origina la rotura \\
8/29/2005 & $6: 30-7 \mathrm{am}$ & Inicio de la inundación \\
8/29/2005 & $7: 30-8 \mathrm{am}$ & Falla del muro del dique \\
8/29/2005 & $9: 00 \mathrm{am}$ & Pico de la tormenta \\
\hline
\end{tabular}

\section{RESULTADOS Y DISCUSIÓN}

\section{Tubificación}

La tubificación es una forma de falla que ocurre cuando el material erosionable se lava debajo del dique, causando inestabilidad. Ocurre cuando el gradiente hidráulico de salida debido a la infiltración excede el gradiente hidráulico crítico del suelo. Este modo de falla se analizó utilizando el programa de análisis de infiltración Seep/W.

a. Consideraciones: El análisis de infiltración utiliza tanto análisis de infiltración de estado estacionario como transiente, con la condición inicial a largo plazo modelada utilizando el estado estacionario, y la marejada ciclónica modelada utilizando un análisis transiente. Debido a que las condiciones están cambiando con el tiempo, un análisis transiente es más preciso. El hidrograma descrito en la sección 2 se usa para modelar el evento de tormenta.

La condición de rotura se modeló para todos los análisis de infiltración utilizando los supuestos descritos en la sección anterior. La excavación del lado de la inundación SM-5 se incluyó en el análisis; sin embargo, tuvo un efecto insignificante en los gradientes de salida calculados.

b. Propiedades del material y condiciones de borde para el análisis de infiltración: Las propiedades del material utilizadas para los análisis de infiltración se dan en las Tablas 2 y 3 . Las condiciones de borde se dan en la Tabla 4. 
Tabla 2. Parámetros de infiltración en estado saturado.

\begin{tabular}{lcccccc}
\hline \multicolumn{1}{c}{ Capa } & $\begin{array}{c}\text { Modelo } \\
\text { (Sat/No saturado) }\end{array}$ & $\begin{array}{c}\text { Ksat } \mathbf{x} \\
(\mathbf{c m} / \mathbf{s e c})\end{array}$ & $\begin{array}{c}\text { Ksat } \mathbf{x} \\
(\mathbf{f t} / \mathbf{s})\end{array}$ & Ky/Kx & $\mathbf{\Theta}_{\text {sat }}$ & $\begin{array}{c}\mathbf{m}_{\mathbf{v}} \\
(\mathbf{1} / \mathbf{p s f})\end{array}$ \\
\hline Material "Shell" & Unsat & $3.05 \mathrm{E}-03$ & $1.00 \mathrm{E}-04$ & 1 & 0.6 & $1.00 \mathrm{E} 05$ \\
Rellenos aguas abajo & Unsat & $1.01 \mathrm{E}-05$ & $3.30 \mathrm{E}-07$ & 1 & 0.5 & $4.00 \mathrm{E} 05$ \\
Excavación (SM-5) & Unsat & $3.05 \mathrm{E}-03$ & $1.00 \mathrm{E}-04$ & 1 & 0.6 & $1.00 \mathrm{E} 05$ \\
Relleno de dique & Unsat & $1.01 \mathrm{E}-05$ & $3.30 \mathrm{E}-07$ & 1 & 0.5 & $1.00 \mathrm{E} 05$ \\
Material del Canal Avenida Jourdan & Unsat & $1.01 \mathrm{E}-07$ & $3.30 \mathrm{E}-09$ & 1 & 0.5 & $1.00 \mathrm{E} 05$ \\
Muro de dique & Sat & $3.05 \mathrm{E}-59$ & $1.00 \mathrm{E}-60$ & 1 & 0 & $1.00 \mathrm{E} 25$ \\
OC superior & Sat & $1.01 \mathrm{E}-05$ & $3.30 \mathrm{E}-07$ & 0.33 & 0.4 & $1.00 \mathrm{E} 05$ \\
OC más bajo & Sat & $1.01 \mathrm{E}-04$ & $3.30 \mathrm{E}-06$ & 0.1 & 0.4 & $1.00 \mathrm{E} 05$ \\
Arcilla Interdistributaria & Sat & $1.01 \mathrm{E}-06$ & $3.30 \mathrm{E}-08$ & 0.5 & 0.5 & $4.00 \mathrm{E} 05$ \\
\hline
\end{tabular}

Tabla 3. Parámetros de infiltración en estado no saturado.

\begin{tabular}{lcccc}
\hline \multicolumn{1}{c}{ Capa } & $\begin{array}{c}\text { Modelo } \\
\text { (Sat/Unsat) }\end{array}$ & $\begin{array}{c}\text { Función de } \\
\text { Permeabilidad } \\
\text { Curva Seep/W }\end{array}$ & \multicolumn{2}{c}{$\begin{array}{c}\text { Función de Permeabilidad } \\
\text { Método }\end{array}$} \\
\hline Material "Shell" & Unsat & Sand & Fredlund & 0.01 \\
Rellenos aguas abajo & Unsat & Clay & Fredlund & 0.21 \\
Excavación (SM-5) & Unsat & Sand & Fredlund & 0.01 \\
Relleno de dique & Unsat & Clay & Fredlund & 0.21 \\
Material del Canal Avenida Jourdan & Unsat & Clay & Fredlund & 0.01 \\
\hline
\end{tabular}

Tabla4. Condiciones de Borde.

\begin{tabular}{ll}
\hline Zona & Condiciones de Borde \\
\hline Zona interior del canal & $\begin{array}{l}\text { Carga total, } \mathrm{H}=0.5 \\
\text { presiones de poro }=0 \text { en } \\
\text { la superficie }\end{array}$ \\
Relleno aguas abajo & $\begin{array}{l}\text { No Flujo, Q=0, } \mathrm{Q}=0, \\
\text { Potencional zona de }\end{array}$ \\
Dique (cresta al pie) & No Flujo, $\mathrm{Q}=0$ \\
Bordes del modelo & No Flujo, $\mathrm{Q}=0$ \\
Borde del muro tablaestaca
\end{tabular}

El gradiente de salida en el punto "A" (Figura 3) fue 0.09 , y el gradiente máximo de salida de 0.26 (punto "B") se encontró en la intersección entre el relleno del dique y el JAC. El gradiente de salida es mayor en el punto B porque la infiltración a través de las arcillas orgánicas se corta y canaliza hacia arriba por el material de baja permeabilidad del JAC.
El gradiente crítico de salida, calculado como ic $=\gamma \mathrm{s} \% \gamma \mathrm{w}$, es ic $=0.76$ para el relleno del dique. Dado que el gradiente máximo de salida es menor que el gradiente crítico de salida, el dique no falla por tubificación. El factor de seguridad para esta condición se da como $\mathrm{F}=0.76 / 0.26=2.9$. Los datos de probabilidad se presentan en la sección de conclusiones.

\section{Estabilidad semi-rotacional}

La inestabilidad semi-rotacional es una falla global de la pendiente que se extiende desde la base de la pared de la tablestaca hasta el pie aguas abajo del dique. La superficie de falla comienza en la base de la pared de la tablestaca debido a la formación de la grieta en el lado de la inundación. Este modo de falla se evaluó utilizando el método de Spencer en el software Slope/W. 


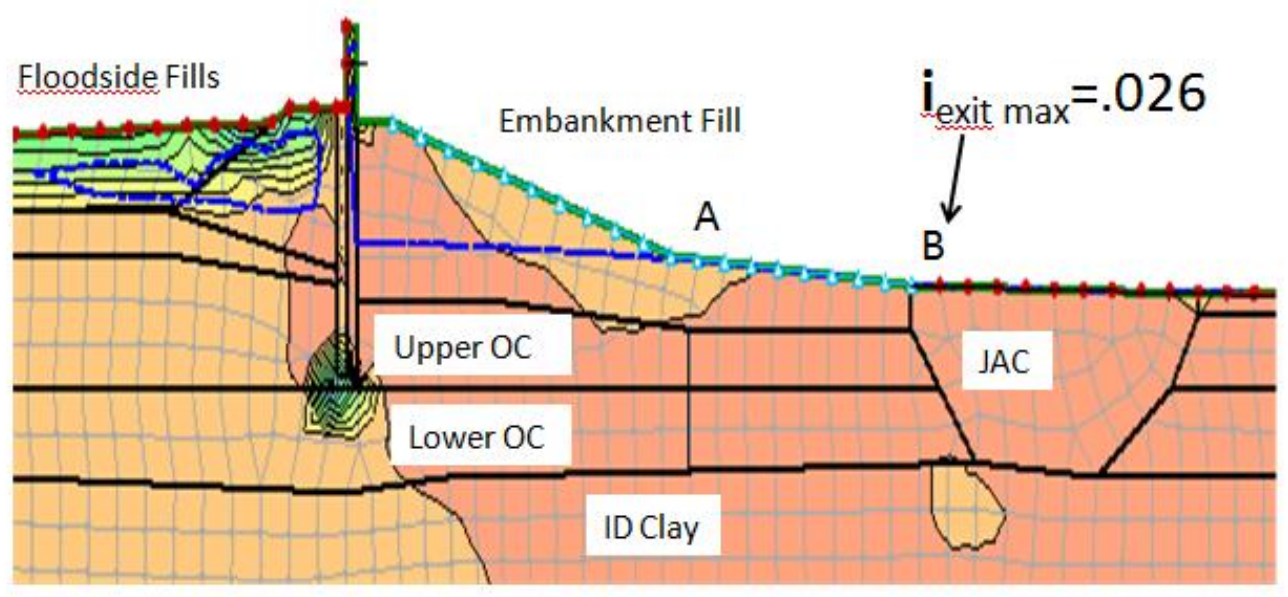

Figura 3: Gradientes hidráulicos verticales cuando la grieta se formó estimados en Seep/W.

\section{Métodos en base a análisis preliminares}

Se determinó que la superficie de falla ocurriría principalmente a través de los materiales de arcilla orgánica. Utilizando el método del factor tiempo (t50) descrito por Duncan and Wright (2005), se determinó que la falla semi-rotacional ocurriría en condiciones no drenadas para todos los valores razonables de mv y kv. También se consideró la erosión producida en el lado de la inundación tuvo un efecto significativo en el modelo de falla semirotacional. Este mecanismo se analizó suponiendo que la falla de la pendiente se inicia en la parte inferior de la pared de la tablestaca y avanzando hacia la base o pie del dique. Las resistencias cortantes de los materiales para el análisis se resumen en la Tabla 5.

Tabla 5. Parámetros de resistencia de los materiales en Slope/W.

\begin{tabular}{|c|c|c|c|c|c|}
\hline \multirow[b]{2}{*}{ Capa } & \multirow[b]{2}{*}{$\begin{array}{c}\text { Peso Específico } \\
\text { (pcf) }\end{array}$} & \multicolumn{2}{|c|}{ No drenada } & \multicolumn{2}{|c|}{ Drenada } \\
\hline & & $\begin{array}{c}\text { Su } \\
\text { (psf) }\end{array}$ & $\mathbf{S u} / \mathbf{p}^{\prime}$ & $\begin{array}{c}\phi ' \\
\text { (degrees) }\end{array}$ & $\begin{array}{c}\mathbf{c}^{\prime} \\
\text { (psf) }\end{array}$ \\
\hline Relleno del dique & 110 & 500 & - & - & - \\
\hline Relleno de tierra & 105 & 500 & - & 30 & 0 \\
\hline UOC (pie del talud) & 105 & 550 & - & - & - \\
\hline UOC (cresta) & 105 & 650 & - & - & - \\
\hline LOC (pie del talud) & 80 & 200 & - & - & - \\
\hline LOC (cresta) & 80 & 300 & - & - & - \\
\hline Arcilla Interdistributaria & 100 & - & 0.23 & - & - \\
\hline
\end{tabular}

Se obtuvo un factor de seguridad de 1,24 para la estabilidad semi-rotacional. La superficie de falla crítica obtenida en Slope/W se muestra en la Figura 4. Los datos de probabilidad se presentan en las conclusiones (sección 4).

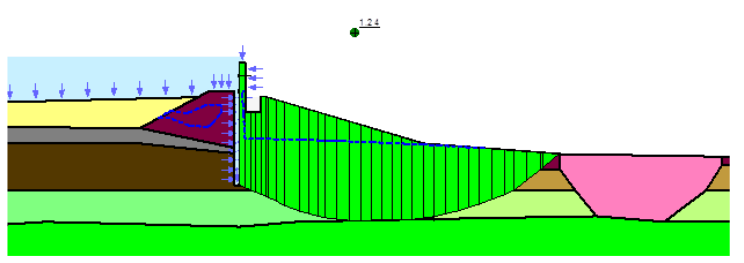

Figura 4. Falla de estabilidad de pendiente semirotacional con FS = 1.24 mostrada. 


\section{Volcamiento debido a la erosión}

Las presiones de tierra de Rankine y las fuerzas hidrostáticas se calcularon en cada lado de la pared de sección I. Se calculó el momento sobre el fondo de la pared de sección I y se comparó para obtener un factor de seguridad. Se asumió que la superficie del agua subterránea se mantiene constante cerca de la elevación 0. Debajo de la superficie del agua, el relleno del dique y la arcilla orgánica se modelan usando condiciones sin drenaje (un análisis de tensión total con $\square^{\prime}=0$ ). Por encima de la superficie del agua, el relleno del dique se modela como un material de fricción sin resistencia a la cohesión. El relleno del dique y la arcilla orgánica son relativamente impermeables, y no es probable que la superficie del agua dentro de ellos cambie drásticamente durante el tiempo de la tormenta.

Efecto de la grieta y la erosión en la estabilidad traslacional y de volcamiento

El análisis supone una grieta completamente formada en el lado de inundación del muro. Por lo tanto, en el lado de la inundación, no hay presiones activas de la tierra y solo una presión hidrostática del agua que lo empuja hacia la pared I. En el lado pasivo, las capas superiores de arcilla orgánica y relleno de diques están actuando como fuerza resistente en la pared.

La socavación aguas debajo de la pared es significativo para este modelo. Sin socavación, el factor de seguridad permanece muy por encima de la unidad. La Figura 7 muestra que el factor de seguridad disminuye con el aumento del nivel de inundación, y continúa disminuyendo a medida que la erosión en el terreno elimina el material de resistencia pasiva. Para que aparezca la grieta del lado de la inundación, debe producirse socavación debido a la acción de las olas antes de completar el sobre embalse del dique. Sin embargo, en este análisis se supone que la mayor parte de la erosión ocurre después del sobreembalse, mientras que el agua fluye libremente sobre la pared.

\section{Erodabilidad}

Briaud et al. (2008) presenta un método para determinar la erosión del suelo en diques. El gráfico, que se muestra en la Figura 6, traza la velocidad de socavación versus la velocidad de la onda. Se determinó que el agua que cae libremente desde la parte superior de la pared hasta el relleno del dique tiene una velocidad de $6,3 \mathrm{~m} / \mathrm{s}$. Con base a esta velocidad, es razonable suponer que se generó aproximadamente 3 pies de socavación en un suelo de erosión promedio entre el momento en que comenzó el desbordamiento total y el momento de la falla.

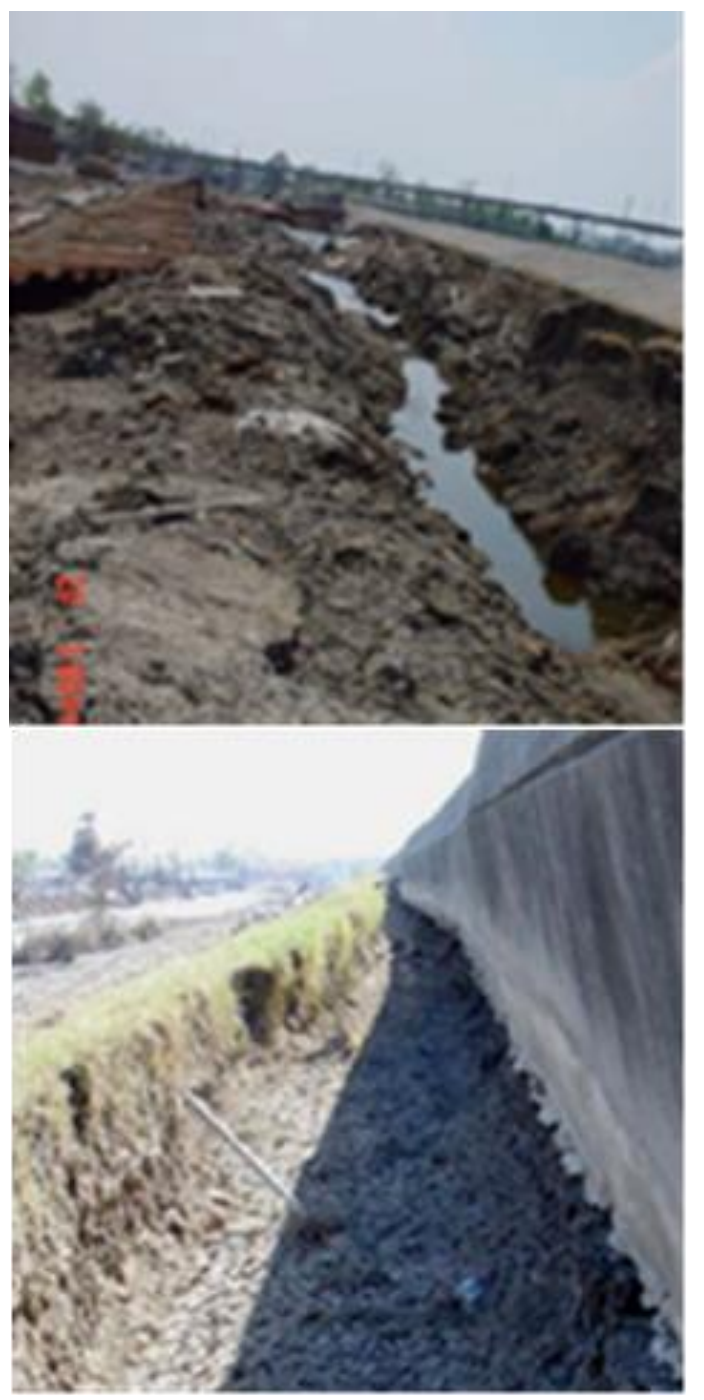

Figura 5. Exceso de erosión en la grieta sur y una sección cercana intacta.

En base a las estimaciones de la erosión observadas en las fotos (Figura 5) y según Briaud et al. (2008) (Figura 6), se calculó que se produjeron 3 pies de socavación debido al sobreembalse. La eliminación de 3 pies de resistencia pasiva produce un $\mathrm{FS}=0.94$ contra el vuelco. La Figura 7 muestra que el factor de seguridad disminuye a medida que aumenta la altura de la tormenta, y a medida que se produce erosión debido al sobreembalse. Los valores de probabilidad se presentan en las conclusiones (sección 4). 


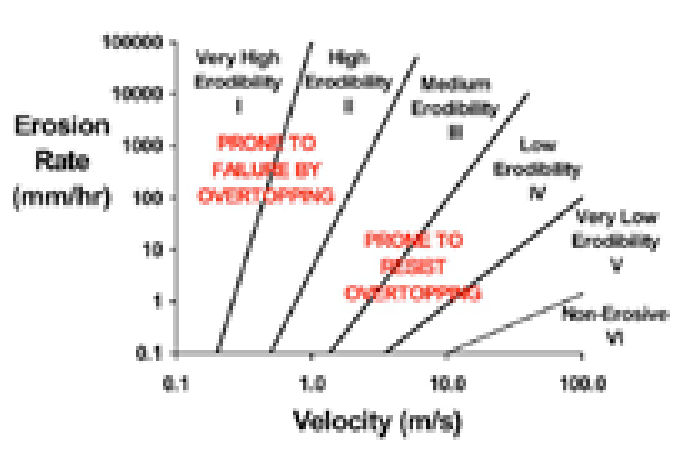

Figura 6. Gráfico de Erodabilidad (Briaud et al., 2008).

\section{Inestabilidad traslacional (deslizante)}

Las presiones de tierra y las presiones de agua en cada lado de la pared I se sumaron para encontrar un factor de seguridad contra la inestabilidad traslacional. Esto modela las fuerzas activas en el lado de la inundación resistidas por las presiones de tierra pasivas. Se utilizaron las mismas condiciones en términos de secuencia de eventos y drenaje que en el análisis de volcamiento. Se calculó que el derrumbe por traslación fue controlado, que tiene un FS $=1.42$ después de 3 pies de socavación. Los resultados se muestran en la Figura 8.

Failure Mode: Overturn

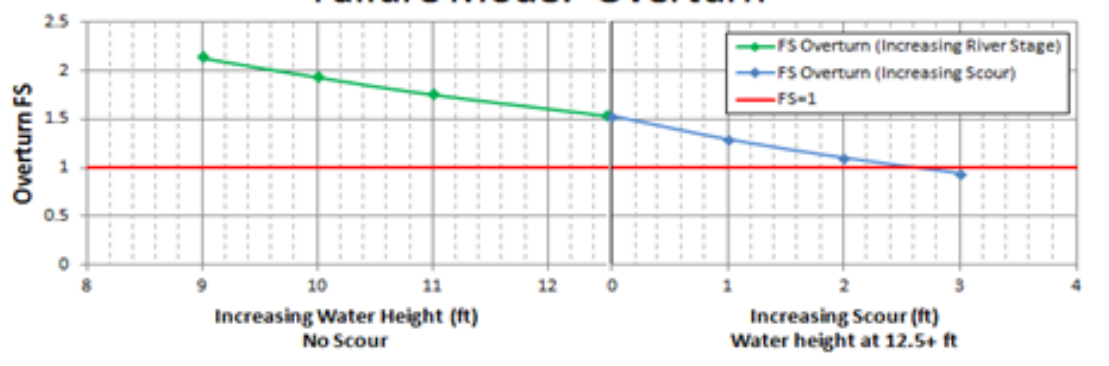

Figura 7. Factor de seguridad contra el modo de falla de volcamiento de la pared I.

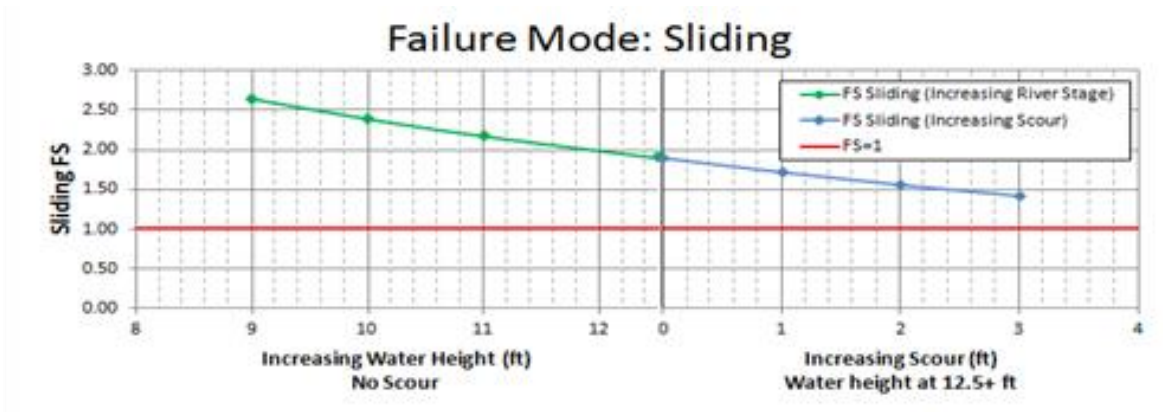

Figura 8. Factor de seguridad contra el modo de falla traslacional de la pared I.

Tabla 6. Factores de seguridad y probabilidad de falla para los modos de falla analizados.

\begin{tabular}{lccc}
\hline Modo de falla & $\mathbf{F}_{\text {MLV }}$ & $\begin{array}{c}\mathbf{V}_{\mathbf{F}} \\
(\boldsymbol{\%})\end{array}$ & $\begin{array}{c}\text { Prob } \\
\text { Falla } \\
(\boldsymbol{\%})\end{array}$ \\
\hline Tubificación & 2.92 & 80 & 12 \\
Falla semi-rotacional (global) & 1.24 & 28 & 24 \\
Volcamiento debido a la erosión & 0.94 & 4 & 88 \\
Estabilidad Traslacional & 1.42 & 3 & 1 \\
\hline
\end{tabular}

Probabilidad de falla a partir de un coeficiente de varianza $(F V)$

Los resultados de la probabilidad de falla a partir de un coeficiente de varianza $(\mathrm{FV})$, planteado en el método de Duncan (2000), se presentan en la Tabla 6. 


\section{CONCLUSIONES}

Basado en los análisis presentados en la sección 3, se determinó que el modo de falla de volcamiento debido a la erosión es el modo crítico de falla. Sin embargo, es posible que otro mecanismo de falla haya sido controlado acorde a los resultados presentados en la tabla 6 , sobre la probabilidad de falla presentada por Duncan (2000). El volcamiento tiene el factor de seguridad más bajo, pero la falla semi-rotacional también tiene un $24 \%$ de probabilidad de ocurrencia. La traslación, o el deslizamiento de la pared, es poco probable que sea el modo crítico de falla porque se ha calculado que el volcamiento ocurre primero la socavación. La tubificación tiene una alta variabilidad porque el análisis es muy sensible a los cambios en la permeabilidad y al coeficiente de cambio de volumen en la arcilla orgánica. A pesar de la gran variabilidad, las tuberías tienen un alto factor de seguridad y no es probable su falla. Todos los análisis han asumido la grieta que origina la inundación. La grieta debe haber ocurrido para causar una falla, caso contrario todos los factores de seguridad excederían la unidad.

Hay varios supuestos y limitaciones a este artículo. Todas las propiedades de ingeniería del suelo utilizadas en los análisis se tomaron de otras fuentes, y no se realizaron pruebas nuevas para este estudio. La precisión de los análisis está limitada no solo por la calidad de la literatura, sino también por el juicio de los autores de las fuentes. Cuando las propiedades de las fuentes no coincidían bien con el criterio de ingeniería de los autores, se seleccionó un nuevo valor, especialmente para el coeficiente de cambio volumétrico mv. Otras limitaciones incluyen haber descontado las fuerzas de las olas en el muro I y no aplicar límites para modelar la lluvia en el lado terrestre del dique. Se usaron factores de presión de Rankine para los análisis de volcamiento y traslación de la pared I, mientras que los métodos más rigurosos pueden encontrar un factor de seguridad más preciso.

\section{Conflictos de intereses}

Los autores firmantes del presente trabajo de investigación declaran no tener ningún potencial conflicto de interés personal o económico con otras personas $\mathrm{u}$ organizaciones que puedan influir indebidamente con el presente manuscrito.

\section{Contribuciones de los autores}

Preparación y ejecución: ACD. ACV; Desarrollo de la metodología: $\mathrm{ACD}$. ACV; Concepción y diseño: ACD. ACV; AL; Edición del artículo: ACD. ACV; Supervisión del estudio: ACD. ACV.

\section{LITERATURA CITADA}

- Bea, R.G. (2008). Engineering Forensic Studies of Performance of the Man-Made Features

- Bordering the Inner Harbor Navigation Canal at the Lower 9th Ward During Hurricane Katrina (Declaration No. II.). Moraga, CA: Risk Assessment and Management Services.

- Briaud, J.L (2008), Levee Erosion by Overtopping in New Orleans during the KatrinaHurricane. ASCE J. Geotech. Geoenviron. Eng. 134 (5). (May 2008).

- Duncan, M., Brandon, T.L., Wright, S.G., \& Vroman, N. (2008). Stability of I-walls in New Orleans during Hurricane Katrina. J. of Geotech. Geoenviron. Eng. 134(5),681-691.

- Duncan, J.M., \& Wright, S.G. (2005). Soil Strength and Slope Stability. Chapter 9: Analyses for Rapid Drawdown, John Wiley and Sons, pp. 151-160.

- Duncan, J.M. (2000). Factors of Safety and Reliability in Geotechnical Engineering. Journal of Geotechnical and Geoenvironmental Engineering, ASCE, 26(4),307-316.

- Dunbar, J.B., Britsch III, L.D. (2008). Geology of the New Orleans Area and the Canal Levee Failures. ASCE J. Geotech. Geoenviron. Eng. (May 2008).

- GEO-SLOPE (2012a). Seepage Modeling with SEEP/W. An Engineering Methodology. Manual de usuario. Edición de julio 2012. GEO-SLOPE International Ltd. Calgary, Canadá. Extraído de: http://www.geo-slope.com en mayo de 2018.

- GEO-SLOPE (2012b). Stability Modeling with SLOPE/W. An Engineering Methodology. Manual de usuario. Edición de julio 2012. GEOSLOPE International Ltd. Calgary, Canadá. Extraído de: http://www.geo-slope.com en mayo de 2018.

- IPET. (2006). Investigation of the Performance of the New Orleans Flood Protection Systems in Hurricane Katrina on August 29, 2005. July 31.

- Rogers, J.D. (2007). "Investigation of Levee Performance in Hurricane Katrina: Levee Failures Along the Inner Harbor Navigation 
Channel. ASCE Geo-Denver 2007 MiniSymposium on Performance Evaluation and Analyses of the New Orleans Levee System in Hurricane Katrina, Denver, Colorado, USA.

- Seed, R.B., Athanasopoulos, A.G, Boutwell, G.P., Bray, J.D., \& Cheung, C. (2007). Investigation of Levee Performance in Hurricane Katrina: The Inner Harbor Navigation Channel. ASCE Geo-Denver. (2007)

- Sills, G.L., Vroman, N.D., Wahl, R.E., \& Schwanz, N.T. (2008). "Overview of New Orleans Levee Failures: Lessons Learned and Their Impact on National Levee Design and Assessment." ASCE J. Geotech. Geoenviron. Eng. 